\title{
Intratumoral delivery of IL-12 gene by polyvinyl polymeric vector system to murine renal and colon carcinoma results in potent antitumor immunity
}

\author{
SK Mendiratta, A Quezada, M Matar, J Wang, HL Hebel, S Long, JL Nordstrom and F Pericle \\ GeneMedicine, Inc, 8301 New Trials Drive, The Woodlands, TX 77381-4248, USA
}

We have utilized a nonviral, polymeric interactive non-condensing (PINC) gene delivery system to deliver IL-12 to two different types of murine tumors, an immunogenic renal cell carcinoma, Renca, and a non-immunogenic colon cell carcinoma, CT26. The delivery of IL-12/polyvinyl pyrrolidone (PVP) complexes into Renca led to the

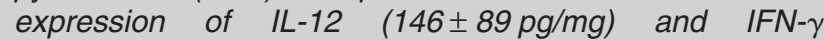
$(160 \pm 82 \mathrm{pg} / \mathrm{mg})$ from explanted tumors in culture supernatants. Treated tumors showed increased infiltration of $\mathrm{NK}, \mathrm{CD} 4^{+}$and $\mathrm{CD} 8^{+} \mathrm{T}$ cells and up-regulation of $\mathrm{MHC}$ class I molecules on leukocytes in both tumors and lymph nodes. Fifty per cent of tumor-bearing mice rejected Renca or CT26 tumors following IL-12/PVP treatments given at optimal doses of 24 and $48 \mu \mathrm{g}$, respectively. While polymorphonuclear cells (PMNs) were partially involved in the development of the antitumor immune response elicited by IL-12/PVP treatment, CD8 ${ }^{+} T$ cells were found to be the primary effectors. In contrast, CD4 $4^{+} T$ cells did not appear to play a significant role in the development of tumor specific immunity. Finally, mice that rejected the tumors following IL-12/PVP treatment were protected against a second challenge with the same tumor. These data provide evidence that a nonviral IL-12 gene delivery system is well tolerated and generates a potent immune response against established tumors.

Keywords: IL-12; gene therapy; immunotherapy; cancer; gene delivery

\section{Introduction}

The goal of cytokine gene-mediated immunotherapy of cancer is to generate a long lasting, tumor-specific, systemic immune response that prevents the establishment of micro-metastases. Cytokines are an important component of the immune system that participate in the development of immune response at multiple levels. They regulate the migration of immunocytes and participate in the activation, maturation and differentiation of the immune response. Cytokine genes can be delivered to specific sites to produce these proteins locally at physiological levels. The local expression of cytokines may be less toxic than their systemic delivery as recombinant proteins. ${ }^{1}$

While a number of cytokines have been tested for immunotherapy of cancer, IL-12 has proven to be one of the most effective in the induction of potent antitumor immunity. ${ }^{2-5}$ IL-12 has numerous immunomodulatory properties, ${ }^{6}$ such as inducing the proliferation of NK and T cells ${ }^{7-9}$ and augmenting their cytolytic activity. ${ }^{9-12}$ Some of the antitumor effects of IL-12 are mediated through the induction of IFN- $\gamma$ from NK cells and T cells. ${ }^{2,3,10,13,14}$ Administration of an IFN- $\gamma$-neutralizing antibody reduces the IL-12-mediated antitumor activity. ${ }^{2}$ Moreover, IFN- $\gamma$ promotes in at least one tumor model (Renca), the secretion of a CXC chemokine, IP-10, which can induce the accumulation of $\mathrm{CD}^{+} \mathrm{T}$ cells in the tumor. ${ }^{15}$ This chemokine is also an anti-angiogenic factor that blocks the growth of tumors by reducing blood supply. ${ }^{16}$ IL-12 is required in the differentiation of T helper cells undergoing antigen priming to Th1 cells ${ }^{17,18}$, which participate in the elimination of intracellular parasites and may help in the development of cytolytic CD8 ${ }^{+} \mathrm{T}$ cell responses against viruses and tumor cells.

Cytotoxicity associated with the systemic use of recombinant IL-12 protein, both in mice ${ }^{19}$ and in humans, ${ }^{20}$ has led researchers to seek alternatives for its delivery to target tissue. Tumor cells or fibroblasts transfected ex vivo with IL-12 ${ }^{21-23}$ effectively induce an antitumor immune response in mouse tumor models. Translation of this system into a clinically acceptable form would involve first deriving stable tumor or fibroblast cell lines from the patient, and then transfecting them with the IL-12 gene followed by re-infusion to the patient. A nonviral gene therapy approach for the treatment of cancer could be an exciting option in the clinic because it would be potentially more efficient and less labor intensive. While viral vectors, such as retrovirus, ${ }^{24}$ vaccinia virus ${ }^{25}$ and adenovirus, ${ }^{26}$ have been successfully used in mouse tumor models, an important issue in bringing them to the clinic is their immunogenicity. Since highly immunogenic viral systems would inhibit repeated use, nonviral systems may hold more promise for the delivery of a specific therapeutic gene into humans.

The gene delivery system used in this study yields a higher level of gene expression in muscle tissues than unformulated DNA. ${ }^{27}$ This system has been used to 
obtain significant biological activity of a variety of transgene products. ${ }^{28,29}$ Using this system to deliver IL-12 into two tumor models - immunogenic (Renca) and less immunogenic (CT26) - we show that up to $50 \%$ of tumorbearing mice in both models reject their tumors with the help of a primarily $\mathrm{CD}^{+} \mathrm{T}$ cell-mediated immune response that has long lasting memory.

\section{Results}

Expression of IL-12 and IFN- $\gamma$ in tumors treated with IL12/PVP

To confirm the expression of IL-12 in vivo, tumors that were previously injected with $50 \mu \mathrm{l}(24 \mu \mathrm{g})$ of IL-12/PVP or empty plasmid (EP)/PVP were explanted 1 day later and cultured as single cell suspensions in complete medium for $24 \mathrm{~h}$. Culture supernatants were separated from cells by centrifugation and assayed for the presence of IL-12 by ELISA. Figure 1a shows that tumors injected with IL-12/PVP, but not EP/PVP, secreted IL-12 $(146 \pm 89 \mathrm{pg} / \mathrm{mg}$ total protein) into their culture super-

a

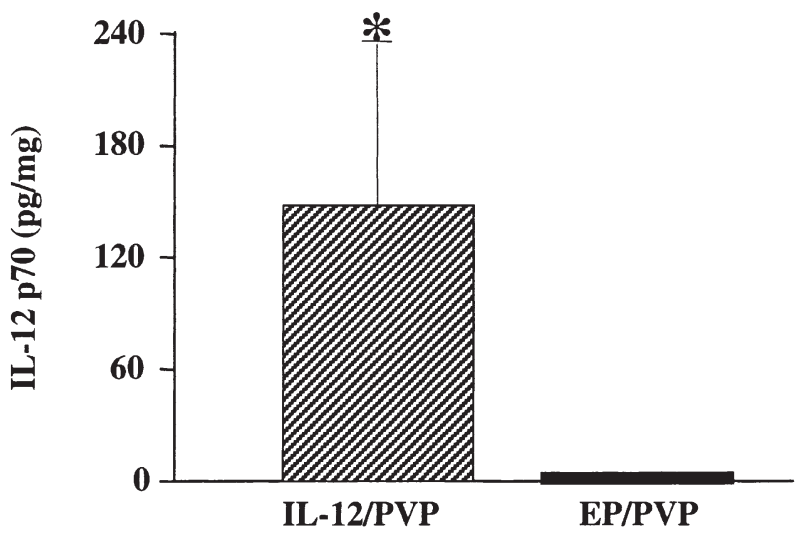

b

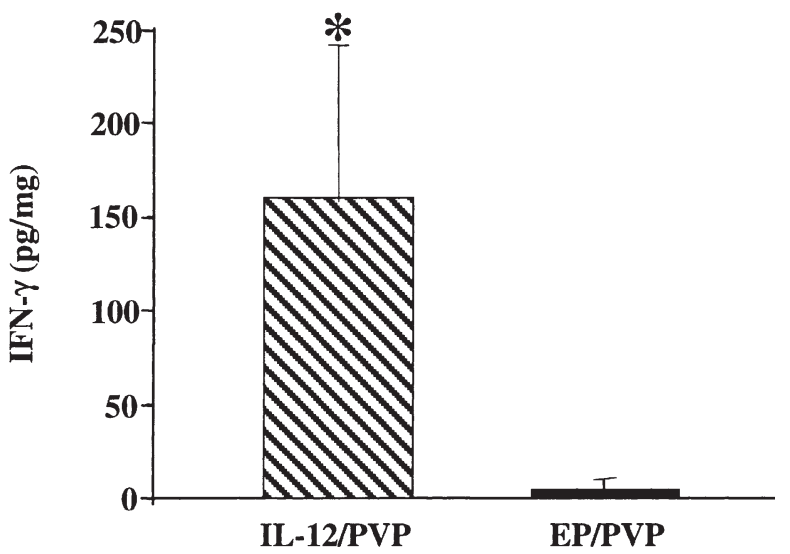

Figure 1 Cytokine production in tumors following IL-12/PVP treatment. Production of IL-12 (a) and IFN- $\gamma(b)$ in Renca tumors. Two groups containing five mice each were treated with a single injection of IL-12/PVP $(24 \mu \mathrm{g})$ or $E P / P V P(24 \mu \mathrm{g})$. One day later, tumors were explanted and single cell suspensions were prepared. Twenty-four hours later culture supernatants were assayed for $I L-12$ and IFN- $\gamma$, and cell pellets were assayed for total protein. Data represent mean values of IL-12 or IFN- $\gamma$ per milligram total protein in each group. ${ }^{*} P<0.05$. natants. These data confirm the in vivo expression capacity of the IL-12/PVP formulation.

IL-12 mediated immunomodulatory properties, such as the development of anti-tumor immunity and the differentiation of a Th1 immune response, are partly mediated by IFN- $\gamma \cdot{ }^{2,3,14}$ To test whether IL-12 produced as a result of the IL-12/PVP treatment could induce the secretion of IFN- $\gamma$, we measured the amounts of IFN- $\gamma$ secreted by explanted tumors as described above. Tumors obtained from mice 1 day after intra-tumoral injection with IL$12 /$ PVP or EP/PVP produced $160 \pm 82$ and $4.6 \pm 5.8 \mathrm{pg}$ IFN- $\gamma / \mathrm{mg}$ total protein, respectively (Figure $1 \mathrm{~b}$ ).

\section{FACS analysis of Renca tumors treated with IL-12/PVP}

IFN- $\gamma$ has been shown to increase the levels of MHC class I and class II molecules on antigen presenting cells ${ }^{30}$ and enhance the proliferation of NK and T cells. ${ }^{7-9}$ To determine if intra-tumoral treatment with IL-12/PVP induced similar effects in the tumor infiltrating cells, FACS analysis of IL-12/PVP-treated Renca tumors was performed. Within 3 days of treatment with IL-12/PVP, increased levels of MHC class I molecules in tumors were observed (Figure 2). There was no detectable change in the levels of MHC class II molecules on tumor infiltrating cells (data not shown). Levels of MHC class I molecules were also increased on cells in the draining lymph nodes (Figure 2). While treatment with the empty plasmid also led to an increase in infiltrating cells (Table 1), the increase was less than that observed with IL-12/PVP. Table I shows that within 3 days of treatment, tumor infiltrating $\mathrm{CD}^{+} \mathrm{T}$ and NK cells in IL-12/PVP-treated tumors increased by approximately four-fold (from $2.79 \%$ to $10.73 \%$ ) and approximately nine-fold (from $0.54 \%$ to $4.87 \%$ ) respectively, when compared with the untreated group. $\mathrm{CD}^{+} \mathrm{T}$ cell increase was found more in the CD8 ${ }^{+}$ $\mathrm{T}$ cell population (approximately eight-fold; from $0.71 \%$ to $5.79 \%$ ) than the $\mathrm{CD} 4^{+} \mathrm{T}$ cell population (approximately three-fold; from $1.92 \%$ to $5.17 \%$ ). The increase in the CD4 ${ }^{+}$ and $\mathrm{CD}^{+} \mathrm{T}$ cell populations was also associated with the expression of the activation marker CD69 on these cells (Table 1). Only a slight increase was observed in numbers of Mac- $1^{\text {hi }}$ cells as a result of treatment with IL-12/PVP (1.5-fold; from $6.93 \%$ to $10.41 \%$ ). Interestingly, treatment with EP/PVP showed a larger increase of Mac- $1^{\text {hi }}$ cells (two-fold; from $6.93 \%$ to $13.82 \%$ ), indicating some biological activity of EP/PVP.

\section{Antitumor effect of IL-12/PVP treatment}

We further analyzed whether IL-12/PVP treatment could induce in vivo an antitumor immune response in Renca and CT26 tumors. Optimal doses of IL-12 expression plasmid were determined for both tumor models by treating them with varying doses of IL-12/PVP (6-48 $\mu \mathrm{g}$ per mouse per treatment). EP/PVP (24 or $48 \mu \mathrm{g}$ ) was used as a control plasmid in these treatments. Each group consisting of six tumor-bearing mice received four treatments (two injections per week for 2 weeks) with IL$12 /$ PVP or EP/PVP and untreated mice were used as control. To determine the optimal dose for treatment with IL-12/PVP, both reduction of tumor size (Figure 3) and rejection of tumors (Table 2) were taken into consideration. The optimal doses of IL-12/PVP treatment for Renca and CT26 were determined to be 24 and $48 \mu \mathrm{g}$ respectively (Table 2 ). 

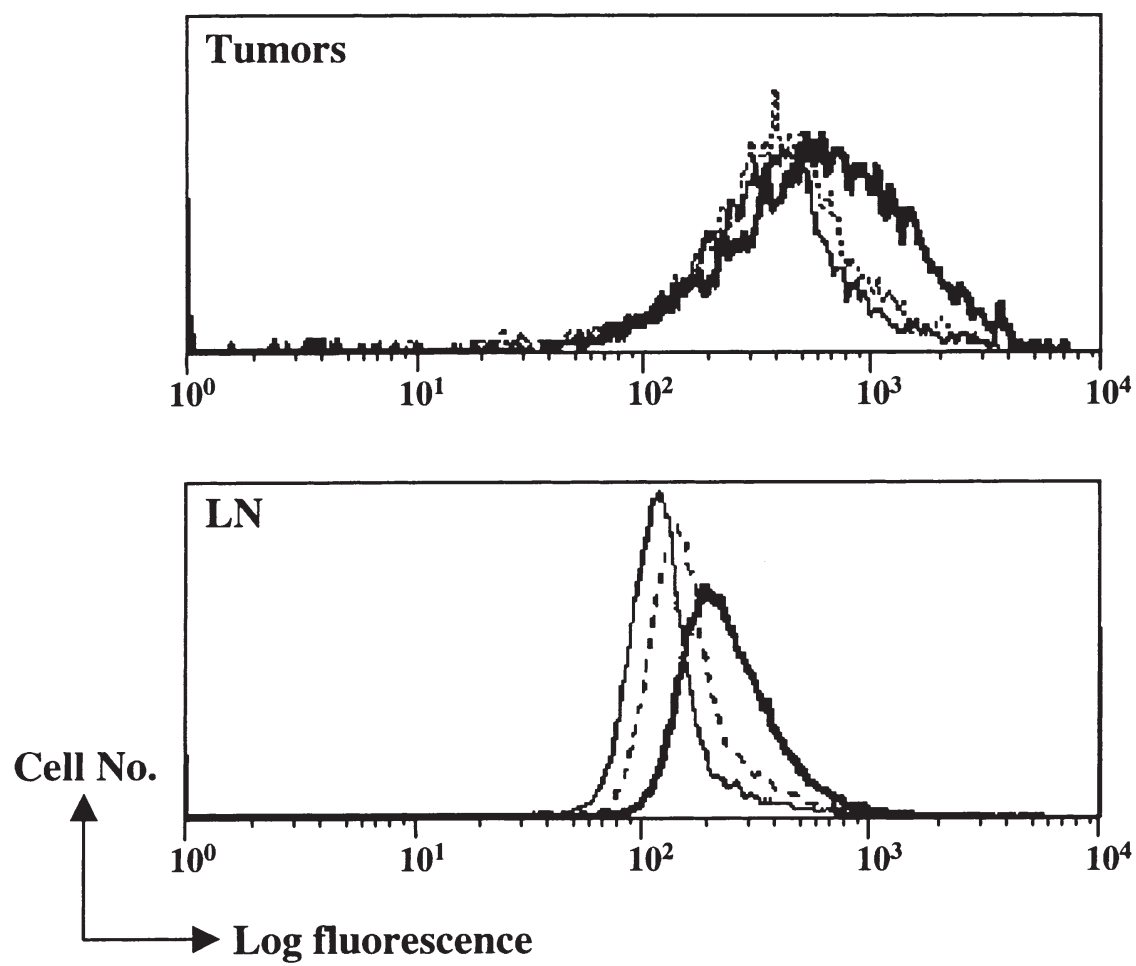

Figure 2 FACS analysis of single cell suspensions obtained from tumors (upper panel) and draining lymph nodes (lower panel). Increased expression of MHC class I in tumors (Tumors) and in draining lymph nodes (LN) of mice treated intra-tumorally with either IL-12/PVP (thick line), EP/PVP (dashed line) or left untreated (thin line).

Table 1 FACS analysis of tumor infiltrating cells ${ }^{a}$

\begin{tabular}{lccc}
\hline & $\begin{array}{c}\text { IL-12/PVP } \\
\%^{b}\end{array}$ & EP/PVP \% & Ctrl \% \\
\hline $\mathrm{CD}^{+} \mathrm{T}$ cells & 10.73 & 4.26 & 2.79 \\
$\mathrm{CD} 4^{+} \mathrm{T}$ cells & 5.17 & 2.87 & 1.92 \\
$\mathrm{CD}^{\circ} 9^{+} \mathrm{CD} 4^{+} \mathrm{T}$ cells & 2.87 & 1.57 & 0.89 \\
$\mathrm{CD} 8^{+} \mathrm{T}$ cells & 5.79 & 1.74 & 0.71 \\
$\mathrm{CD} 69^{+} \mathrm{CD} 8^{+} \mathrm{T}$ cells & 4.37 & 1.11 & 0.45 \\
$\mathrm{NK}$ cells & 4.87 & 1.33 & 0.54 \\
Mac-1 ${ }^{\text {hi }}$ cells & 10.41 & 13.82 & 6.93 \\
\hline
\end{tabular}

${ }^{a}$ Numbers and types of tumor infiltrating cells were analyzed 3 days following IL-12/PVP or EP/PVP treatment and compared with untreated mice (ctrl). Data represented have been confirmed in two independent experiments.

bPercentage of total cells in the tumor.

\section{Tumor rejection induced by IL-12/PVP is predominantly} $C D 8^{+} T$ cell mediated

To study the role of individual populations of the immune system in inducing IL-12-mediated antitumor immune response, mice were depleted of granulocytes/polymorphonuclear (PMN), $\mathrm{CD}^{+} \mathrm{T}$ or $\mathrm{CD}^{+} \mathrm{T}$ cells using monoclonal antibodies. Rejection of Renca and CT26 tumors induced by IL-12/PVP was completely abrogated in mice depleted of $\mathrm{CD}^{+} \mathrm{T}$ cells (Figure 4). In contrast, depletion of $\mathrm{CD}^{+} \mathrm{T}$ cells did not affect tumor rejection following IL-12/PVP treatment of CT26 tumors (80\% versus $80 \%$ ) and Renca tumors $(100 \%$ versus $80 \%$ ).

\section{IL-12/PVP-mediated tumor-specific immunity is associated with memory}

We wanted to investigate whether mice that had rejected the primary tumors as a result of treatment with IL12/PVP had developed specific antitumor immune memory. Mice that rejected Renca or CT26 following IL12 /PVP treatment, were re-challenged subcutaneously with fresh tumor cells in the opposite flank (right) 40-50 days after the first tumor challenge (left flank). More than $50 \%$ of mice that had rejected the primary tumors as a result of cytokine gene treatment did not develop any secondary tumor. In contrast naive mice injected with either Renca or CT26 readily developed tumors (Table 2).

\section{Discussion}

In this article we demonstrate that delivery of IL-12 gene by a nonviral vector system to established tumors induced a potent antitumor immune response. Treatment of tumor-bearing mice with an IL-12 expression plasmid formulated with PVP led to tumor rejection, which was primarily mediated by $\mathrm{CD}^{+} \mathrm{T}$ cells.

Administration of immunomodulatory cytokines is an important strategy for generating immune responses against cancer. However, systemic delivery of cytokines for the treatment of cancer in mice and humans is associated with toxic side-effects. ${ }^{19,20}$ This limitation created a need for alternative approaches, such as the delivery of cytokine genes to the site of the tumor, ${ }^{24-26}$ that reduce toxicity while maintaining the benefits. Various vectors have been successfully utilized to deliver IL-12 genes to the site of the tumor, eg, retrovirus, ${ }^{24}$ adenovirus ${ }^{26}$ and vaccinia virus. ${ }^{25}$ However, these viral systems are labor 

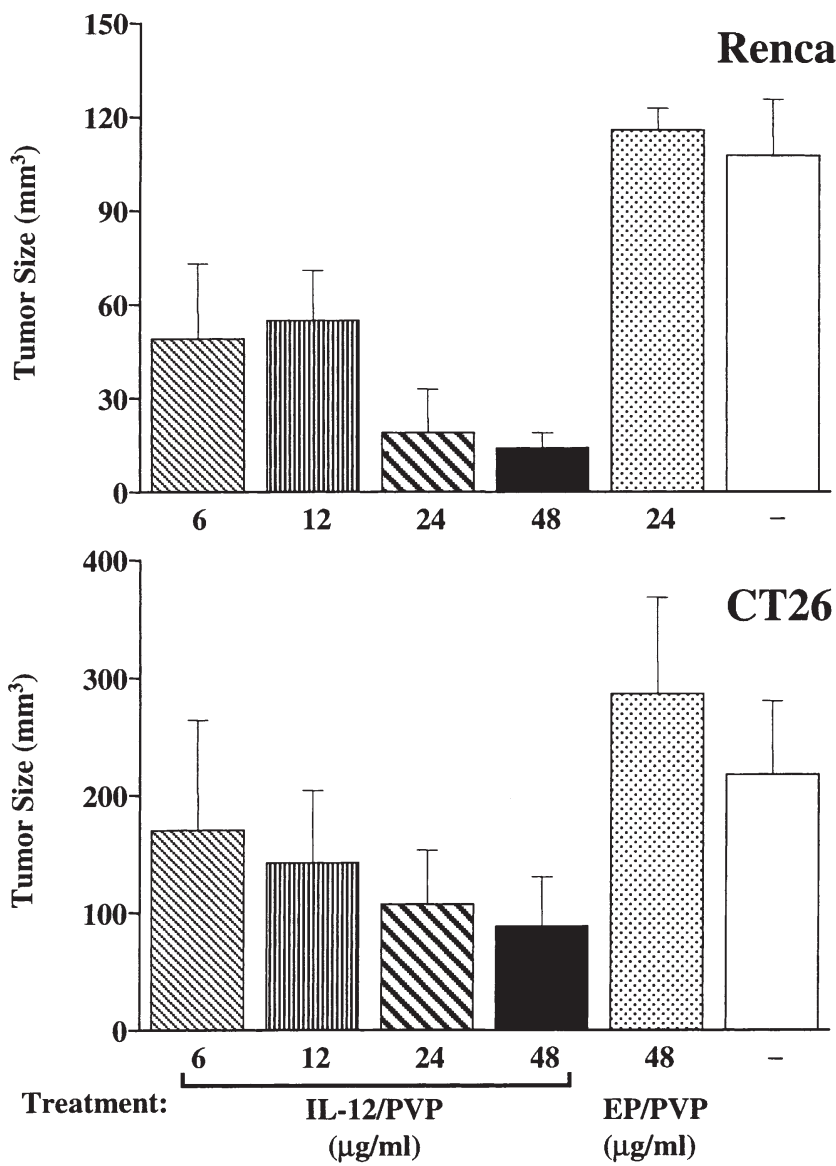

Figure 3 Reduction of tumor size following treatment with IL-12/PVP. Mice bearing Renca or CT26 tumors were divided into groups of six each and treated with varying doses of IL-12/PVP $(6,12,24$ or $48 \mu \mathrm{g})$, $E P / P V P(48 \mu \mathrm{g})$ or untreated. These treatments were repeated twice every week for 2 weeks (total of four injections). Tumor size was measured before each treatment. Data represent mean values of tumor size in each group on day 17 and 16 for Renca and CT26, respectively.

intensive, are associated with immunogenicity of the vector, and bear the potential danger of developing infectious agents. Nonviral vectors provide an important and potentially safer alternative. A nonviral, polymeric, interactive, non-condensing, (PINC) gene delivery system used in our study consistently results in a greater amount of gene expression from muscle tissues as compared with unformulated plasmid DNA. ${ }^{27}$ Recently, intra-muscular expression of biologically active human IGF-I and factor IX have also been demonstrated in vivo using this system. ${ }^{28,29}$ To assess the immunogenicity of plasmid formulated in PINC, we assayed for the presence of anti-plasmid antibodies in serum of dogs repeatedly injected with this formulation and did not detect any antibodies to the administered plasmid (unpublished data). Furthermore, we observed that delivery of our expression system for IL-12 lacks any obvious toxicity such as loss of weight or any signs of distress, that has been reported in mice following systemic delivery of IL-12 protein. $^{31}$

The mechanism of action of the IL-12/PVP delivery system is consistent with the proposed mode of action of IL-12 described by other groups using IL-12 recombinant protein. Several studies have shown that IL-12 mediates its antitumor functions partly through IFN- $\gamma^{2,3,14,32}$ In

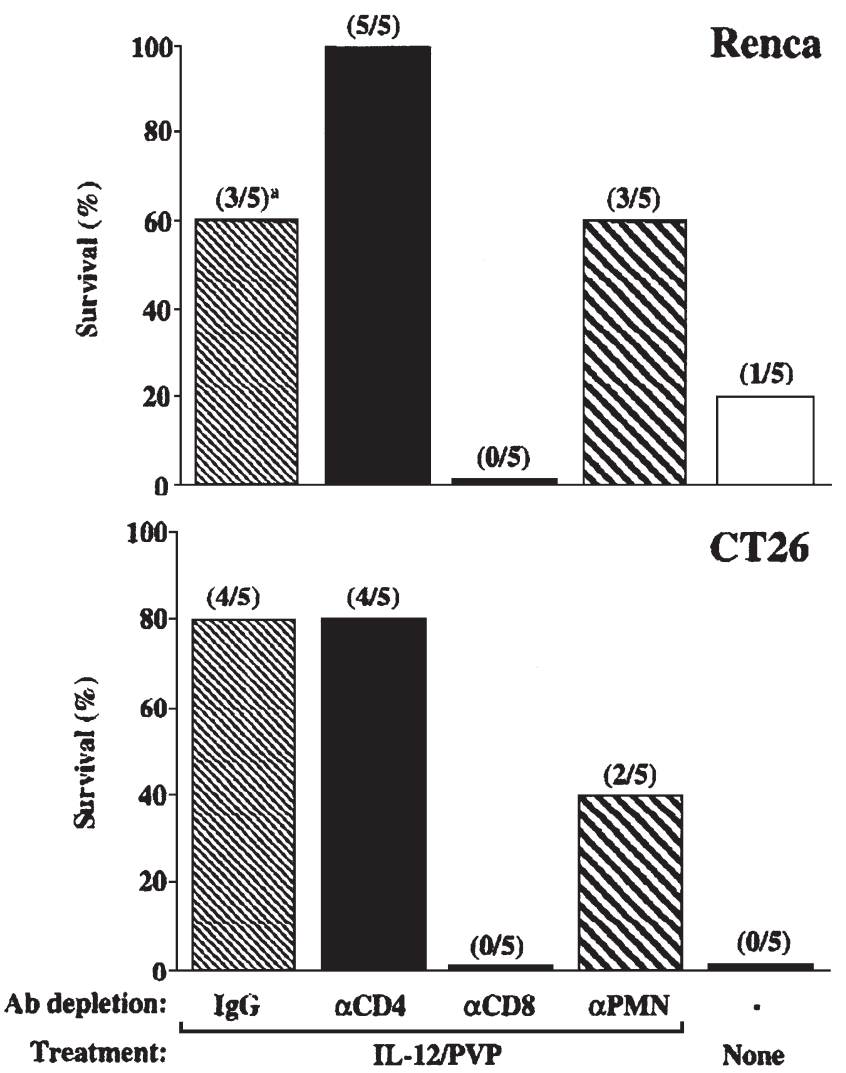

Figure 4 Role of individual cells of the immune system in the development of immune response against tumors. As described in Materials and methods, mice that were depleted of $P M N, C D 4^{+}$or $C D 8^{+} T$ cells by injection of monoclonal antibodies, were treated twice every week for 2 weeks with IL-12/PVP (Renca, $24 \mu \mathrm{g}$ and CT26, $48 \mu \mathrm{g}$ ). Data represent the percentage of mice in each group that rejected the tumors completely. ${ }^{a}$ Mice rejecting tumors per total mice challenged. 
conjunction with IFN- $\gamma$, IL-12 induces the differentiation of a Th1 immune response, ${ }^{17,18}$ which may promote the establishment of an antitumor immune response through increased IFN- $\gamma$ production or by conditioning dendritic cells to activate antigen-specific $\mathrm{CD} 8^{+} \mathrm{T}$ cells. ${ }^{33}$ Local production of IL-12 using the IL-12/PVP delivery system, resulted in increased expression of IFN- $\gamma$ in the tumor (32-fold) and in the draining lymph nodes (data not shown). Within 3 days of treatment with IL-12/PVP, we also observed an increased level of MHC class I molecules on cells in tumors and lymph nodes, which may be a result of elevated levels of IFN- $\gamma$ in those tissues (Figure 2). At the same time, we also noticed an influx of tumor infiltrating $\mathrm{NK}, \mathrm{CD} 4^{+} \mathrm{T}$ and $\mathrm{CD}^{+} \mathrm{T}$ cells (Table 1 ). Most of the CD8 ${ }^{+} \mathrm{T}$ cells also expressed CD69, a marker expressed by activated $\mathrm{T}$ cells which may indicate an augmented cytolytic activity. Activated CD8 ${ }^{+} \mathrm{T}$ cells may be better equipped to kill tumor cells. The increased infiltration of NK and activated $\mathrm{T}$ cells was observed even after 3-4 days of a second IL-12/PVP treatment and was associated with further reduction of tumor size (data not shown). While treatment of tumors with EP/PVP also induced an influx of immune cells, tumors treated with IL-12/PVP had a significantly higher infiltration. The influx of immune cells induced by EP/PVP could be a nonspecific effect due to CpG motifs in the plasmid, ${ }^{34}$ or a result of inflammation induced by the multiple injections required to treat the tumor.

Although PMNs were found to be important in the development of a robust immune response, $\mathrm{CD} 8^{+} \mathrm{T}$ cells were the primary effectors of antitumor immunity induced by IL-12/PVP. Mice depleted of these cells no longer responded to IL-12/PVP treatment (Figure 4). In contrast, $\mathrm{CD}^{+} \mathrm{T}$ cells were either not required in the development of antitumor immunity, as seen in the CT26 tumor model, or as in Renca, appeared to suppress its development. $\mathrm{CD}^{+} \mathrm{T}$ cells have been previously shown to prevent the infiltration of $\mathrm{CD}^{+} \mathrm{T}$ cells into the tumor, rather than suppressing their activation. ${ }^{35}$ The development of tumor-specific CD8 ${ }^{+} \mathrm{T}$ cell effectors was associated with a long lasting memory, since mice that rejected the primary Renca and CT26 tumors were resistant to a subsequent challenge of fresh tumor cells (Table 2).

It has been reported that the amount of IL-12 available at the site of the tumor is a determinant of tumor regression. ${ }^{36}$ In a C26 colon carcinoma tumor model, systemic delivery of recombinant IL-12 protein was unable to inhibit tumor growth, because effective levels of IL-12 at the tumor site could not be generated without exceeding the systemic levels that were toxic. Although the nonviral vector system used in this study produced levels of IL-12 (pg) in tumors that were lower than those obtained with viral vectors (ng), the amount generated was sufficient to induce a potent antitumor immune response. Moreover, mice treated with EP/PVP did not develop antitumor immunity against Renca or CT26 suggesting that tumor regression observed in IL-12/PVPtreated mice is indeed IL-12 gene dependent.

This study shows that intra-tumoral delivery of IL-12 genes using a polyvinyl polymeric delivery system is an effective method of generating antitumor immunity. Local production of IL-12 following this gene therapy approach led to tumor regression and rejection which was not associated with toxicity. Thus, this gene delivery/expression system may be applicable to delivering IL-12 to patients for the treatment of potentially metastatic tumors, such as head and neck cancer and melanoma, where primary lesions are easily accessible by direct intra-tumoral injection.

\section{Materials and methods}

\section{Construction and formulation of cytokine gene expression plasmids}

The expression plasmid for mouse IL-12 (pIN0961) is described separately. ${ }^{37}$ Briefly, the p35 and p40 subunits of IL-12 are expressed from two independent transcription units that were incorporated into a single plasmid. Each transcription unit was driven by the human cytomegalovirus immediate-early enhancer/promoter, contained a synthetic intron in its $5^{\prime}$ untranslated region and had its $3^{\prime}$ end defined by the bovine growth hormone poly (A) signal. An expression plasmid with the CMV enhancer/promoter and bovine growth hormone poly (A) signal, but no coding region, served as the empty plasmid control. Plasmids were grown in $\mathrm{DH} 5 \alpha$ E. coli and purified by alkaline lysis and proprietary chromatographic methods (GeneMedicine, Inc.). Purified plasmid utilized for intra-tumoral injections had low levels of endotoxin ( $<20$ endotoxin unit/mg). Purified plasmids were formulated with a $5 \%$ PVP solution ${ }^{27}$ to generate IL-12/PVP and EP/PVP formulations.

\section{Tumor cell lines}

Renca, an immunogenic, spontaneously arising murine renal cell carcinoma, and CT-26, a non-immunogenic, colon adenocarcinoma, were kind gifts of Dr Drew M Pardoll (Johns Hopkins Hospital, Baltimore, MD, USA). Tumor cells were grown in RPMI 1640 medium which was supplemented with $10 \%$ FBS, $100 \mathrm{U} / \mathrm{ml}$ penicillin, $100 \mathrm{U} / \mathrm{ml}$ streptomycin and $50 \mu \mathrm{g} / \mathrm{ml}$ gentamycin (all from Life Technologies, Gaithersburg, MD, USA) at $37^{\circ} \mathrm{C}$ and humidified $5 \% \mathrm{CO}_{2}$.

\section{Mice}

Eight-week-old female BALB/c mice were purchased from Harlan Laboratories (Houston, TX, USA). Mice were maintained on ad libitum rodent feed and water at $23^{\circ} \mathrm{C}$, $40 \%$ humidity, and a $12 \mathrm{~h} / 12 \mathrm{~h}$-light/dark cycle. All mice were acclimated for at least 4 days before the start of the study. All studies were performed in accordance with generally acceptable animal use guidelines.

\section{Tumor implantation and treatment}

To generate tumors, BALB/c mice were subcutaneously implanted in the middle of the left flank with $30 \mu$ of a single cell suspension containing either $7 \times 10^{5}$ Renca cells or 1 x $10^{5}$ CT2 6 cells. Tumor volume was measured with an electronic caliper by measuring its two perpendicular diameters and its depth. Treatment of the tumors was started after about 7 days when they reached a size of $8-10 \mathrm{~mm}^{3}$. Tumors were treated with different plasmid formulations in volumes of $50 \mu \mathrm{l}$ each, at 3-4 day intervals for a total of four treatments in 2 weeks. Tumor sizes were measured twice a week for a period of 40-50 days. In both challenge and re-challenge studies, mice that developed tumors larger than $1 \mathrm{~cm}^{3}$ were killed for humane reasons. 
Renca tumor-bearing mice were injected intra-tumorally with IL-12/PVP or EP/PVP. Tumors were obtained from these mice $24 \mathrm{~h}$ later and single cell suspensions from these were incubated in culture at $37^{\circ} \mathrm{C}$ for $24 \mathrm{~h}$. Supernatants were separated from the cells by centrifugation. Standard sandwich ELISA kits from Genzyme (Cambridge, MA, USA) were used to measure IL-12 and IFN- $\gamma$ in the culture supernatants, as per the manufacturer's instructions. Measurement of total protein in the cell pellets was carried out by first treating them with lysis solution $(10 \mathrm{~mm}$ Tris- $\mathrm{HCl}, 1.0 \mathrm{~mm}$ EDTA, $0.1 \mathrm{~m}$ $\mathrm{NaCl}, 0.5 \%$ Triton $\mathrm{X}-100)$ in the presence of a protease inhibitor cocktail from Boehringer-Mannheim (Mannheim, Germany) and then homogenizing for $5 \mathrm{~min}$ at $4^{\circ} \mathrm{C}$ using a mini bead-beater (Sunon, Taiwan). Supernatants collected by centrifuging the samples for $5 \mathrm{~min}$ at $7500 \mathrm{~g}$, were used for protein quantification using BCA protein assay kit from Pierce (Rockford, IL, USA). Cytokine levels per milligram of total protein were measured for each sample.

\section{In vivo antibody-mediated depletion of leukocytes}

All antibody treatments were performed twice. The first injection of the antibody was given intravenously 1 day before starting the cytokine gene treatment. This was followed by a second antibody injection of the same concentration, given intraperitoneally, 1 day before the third injection of cytokine treatment. All cell type-specific antibodies were injected in a volume of $500 \mu \mathrm{l}$. Ascites of the following antibodies were used at appropriate dilutions/concentrations - anti-CD4 (GK1.5, TIB207; ATCC, Rockville, MD, USA), 1:10; anti-CD8 (2.43, TIB210, ATCC), 1:100; anti-asialo GM1 (anti-NK cells) (Wako, Osaka, Japan) $50 \mu \mathrm{l}$; and anti-GR-1 (anti-PMNs) (RB68C5; Pharmingen, San Diego, CA, USA) $100 \mu \mathrm{g}-$ and were determined to be sufficient to maintain depletion of specific cell subsets for the duration of the experiment (data not shown).

\section{Flow cytometric analysis of tumor infiltrating cells}

Single cell suspensions of tumors were made by gently grinding the tumor between the frosted ends of two glass slides in $5 \mathrm{ml}$ of Hank's balanced salt solution (Life Technologies) containing collagenase (Sigma, St Louis, $\mathrm{MO}, \mathrm{USA}$ ) at a concentration of $0.28 \mathrm{mg} / \mathrm{ml}$. This suspension was then transferred to a $50 \mathrm{ml}$ Falcon tube containing 10-15 silica beads of approximately $2.5 \mathrm{~mm}$ diameter (Biospec Products, Bartlesville, OK, USA) and shaken in a $37^{\circ} \mathrm{C}$ water bath for $30 \mathrm{~min}$. Enzyme action was stopped by the addition of $40 \mathrm{ml}$ of phosphate-buffered saline (PBS; Life Technologies) containing 1\% FBS. Cells were passed through a $70 \mu \mathrm{m}$ nylon strainer (Falcon, Lincoln Park, NJ, USA) to remove any debris. Cells were washed three times before staining with various antibodies for FACS analysis. Before staining with various antibodies, cells were stained with anti-CD16/32 antibody to block the Fc $\gamma \mathrm{II} / \mathrm{III}$ receptors. Based on staining with various leukocyte-specific antibodies and forward- and side-scatter analysis, cells in the tumor cell suspension could be gated into different groups of infiltrating cell populations which were further compared between various groups of treatment. For the staining of NK cells, only cells that were positive for staining with the pan NK cell antibody, DX5, and negative for the T cell marker, CD3, were con-

sidered. FACS analysis was performed on FACSCalibur (Becton Dickinson, San Jose, CA, USA).

\section{Antibodies}

The following antibodies were all obtained from Pharmingen. The fluorescein isothiocyanate (FITC)-labeled antiCD3 antibody 145-2C11 (armenian hamster IgG, group 1), the FITC-labeled anti-CD4 antibody GK1.5 (IgG2b), the FITC-labeled anti-CD8 antibody 53-6.7 (IgG2a), the phycoerythrin (PE)-labeled anti-CD69 antibody H1.2F3 (armenian hamster IgG, group 1), the PE-labeled pan NK cell antibody DX5 (rat IgM), the FITC-labeled anti-H-2D ${ }^{d}$ antibody 34-2-12 (mouse IgG2a), and the unlabeled antiCD16/32 antibody 2.4G2 (rat IgG2b). The PE-labeled anti-CD11b (Mac-1) antibody M1/70.15 (rat IgG2b) was obtained from Caltag (San Francisco, CA, USA).

\section{Statistical analyses}

The non-parametric Mann-Whitney $U$ statistic was used to analyze cytokine ELISA data. Tumor rejection data was analyzed by cross-tabulation analysis using the $\chi^{2}$ test.

\section{Acknowledgements}

We thank Dr Michael Fons for helpful discussions and critical review of the manuscript, Dorothy Reimer and Ram Mahato for help in establishing procedure to make single cell preparation from tumors. Integrated Manufacturing and Analytic Teams at GeneMedicine, Inc. contributed significantly to this study.

\section{References}

1 Mier JW, Atkins MB. Mechanisms of action and toxicity of immunotherapy with cytokines. Curr Op Oncol 1993; 5: 10671072.

2 Brunda MJ et al. Role of interferon- $\gamma$ in mediating the antitumor efficacy of interleukin-12. J Immunother 1995; 17: 71-77.

3 Nastala CL et al. Recombinant IL-12 administration induces tumor regression in association with IFN-gamma production. J Immunol 1994; 153: 1697-1706.

4 Rakhmilevich AL et al. Cytokine gene therapy of cancer using gene gun technology: superior antitumor activity of interleukin12. Hum Gene Ther 1997; 8: 1303-1311.

5 Cavallo $\mathrm{F}$ et al. Antitumor efficacy of adenocarcicoma cells engineered to produce interleukin 12 (IL-12) or other cytokines compared with exogenous IL-12. J Natl Cancer Inst 1997; 89: 10491058.

6 Trinchieri G. Interleukin-12: a proinflammatory cytokine with immuno-regulatory functions that bridge innate resistance and antigen-specific adaptive immunity. Ann Rev Immunol 1995; 13: 251-276.

7 Gately MK et al. Regulation of human lymphocyte proliferation by a heterodimeric cytokine, IL-12 (cytotoxic lymphocyte maturation factor). I Immunol 1991; 147: 874-882.

8 Perussia B et al. Natural killer (NK) cell stimulatory factor or IL12 has differential effects on the proliferation of TCR- $\alpha \beta+$, TCR$\gamma \delta+$ T lymphocytes and NK cells. J Immunol 1992; 149: 3495-3502.

9 Robertson MJ et al. Response of human natural killer (NK) cells to NK cell stimulatory factor (NKSF): cytolytic activity and proliferation of NK cells are differentially regulated by NKSF. J Exp Med 1992; 175: 779-788.

10 Kobayashi $\mathrm{M}$ et al. Identification and purification of natural killer cell stimulatory factor (NKSF), a cytokine with multiple biologic effects on human lymphocytes. J Exp Med 1989; 170: $827-845$. 
11 Naume B, Gately MK, Espevik TA. Comparative study of IL-12 (cytotoxic lymphocyte maturation factor)-, IL-2, and IL-7induced effects on immunomagnetically purified CD56+ NK cells. J Immunol 1992; 148: 2429-2436.

12 Gately MK, Wolitzky AG, Quinn PM, Chizzonite R. Regulation of human cytolytic lymphocyte responses by interleukin-12. Cell Immunol 1992; 143: 127-142.

$13 \mathrm{Chan} \mathrm{SH}$ et al. Induction of interferon gamma production by natural killer cell stimulatory factor: characterization of the responder cells and synergy with other inducers. J Exp Med 1991; 173:869-879.

14 Brunda MJ et al. Antitumor and antimetastatic activity of interleukin 12 against murine tumors. J Exp Med 1994; 178: 1223-1230.

15 Tannenbaum CS et al. Cytokine and chemokine expression in tumors of mice receiving systemic therapy with IL-12. J Immunol 1996; 156: 693-699.

16 Angiolillo AL et al. Human interferon-inducible protein 10 is a potent inhibitor of angiogenesis in vivo. J Exp Med 1995; 182: 155-162.

17 Hsieh CS et al. Development of TH1 CD4+ T cells through IL12 produced by Listeria-induced macrophages. Science 1993; 260: 547-549.

18 Manetti R et al. Natural killer cell stimulatory factor (interleukin 12 [IL-12]) induces $\mathrm{T}$ helper type (Th1)-specific immune responses and inhibits the development of IL-4 producing Th cells. J Exp Med 1993; 177: 1199-1204.

19 Orange JS et al. Mechanism of interleukin 12-mediated toxicities during experimental viral infections: role of tumor necrosis factor and glucocorticoids. J Exp Med 1995; 181: 901-914.

20 Marshall E. Cancer trial of interleukin-12 halted. Science 1995; 268: 1555.

21 Zitvogel L et al. Cancer immunotherapy of established tumors with IL-12: effective delivery by genetically engineered fibroblasts. J Immunol 1995; 155: 1393-1403.

22 Tahara $\mathrm{H}$ et al. Fibroblasts genetically engineered to secrete interleukin 12 can suppress tumor growth and induce antitumor immunity to a murine melanoma in vivo. Cancer Res 1994; 54: 182-189.

23 Tahara $\mathrm{H}$ et al. Effective eradication of established murine tumors with IL-12 gene therapy using a polycistronic retroviral vector. J Immunol 1995; 154: 6466-6474.

24 Zitvogel L et al. Construction and characterization of retroviral vectors expressing biologically active human interleukin-12. Hum Gene Ther 1994; 5: 1493-1506.
25 Meko JB, Yim JH, Tsung K, Norton JA. High cytokine production and effective antitumor activity of a recombinant vaccinia virus encoding murine interleukin 12. Cancer Res 1995; 55: 4765-4770.

26 Bramson JL et al. Direct intratumoral injection of an adenovirus expressing interleukin-12 induces regression and long-lasting immunity that is associated with highly localized expression of interleukin-12. Hum Gene Ther 1996; 7: 1995-2002.

27 Mumper RJ et al. Polyvinyl derivatives as novel interactive polymers controlled gene delivery to muscle. Pharmac Res 1996; 5 701-709.

28 Alila $\mathrm{H}$ et al. Expression of biologically active human insulinlike growth factor-I following intramuscular injection of a formulated plasmid in rats. Hum Gene Ther 1997; 8: 1785-1795.

29 Anwer K et al. Systemic effect of human growth hormone after intramuscular injection of a single dose of a muscle-specific gene medicine. Hum Gene Ther 1998; 9: 659-670.

30 Rosa FM, Cochet MM, Fellous M. Interferon and major histocompatibility complex genes: a model to analyse eukaryotic gene regulation? Interferon 1986; 7: 48-87.

31 Tare NS, et al. Administration of recombinant interleukin-12 to mice suppresses hematopoiesis in the bone marrow but enhances hematopoiesis in the spleen. J Interfer Cytok Res 1995; 15: $377-383$.

32 Fujiwara H, Hamoaka T. Antitumor and antimetastatic effects of interleukin 12. Cancer Chemother Pharmacol 1996; 38 (Suppl.): S22-S26.

33 Ridge JP, Rosa FD, Matzinger P. A conditioned dendritic cell can be a temporal bridge between a CD4+ T-helper and a Tkiller cell. Nature 1998; 393: 474-478.

34 Freimark BD et al. Cationic lipids enhance cytokine and cell influx levels in the lung following administration of plasmid: cationic lipid complexes. J Immunol 1998; 160: 4580-4586.

35 Martinotti A et al. CD4 T cells inhibit in vivo the CD8-mediated immune response against murine colon carcinoma cells transduced with interleukin-12 genes. Eur J Immunol 1995; 25: 137146.

36 Colombo MP et al. Amount of interleukin 12 available at the tumor site is critical for tumor regression. Cancer Res 1996; 56 : 2531-2534.

\section{Reference added in proof}

37 Blezinger $\mathrm{P}$ et al. Intra-tracheal administration of IL-12 plasmid/cationic lipid complexes inhibits murine lung metastases. Hum Gene Ther (in press). 